\title{
Statistical detection of faults in swarm robots under noisy conditions
}

\author{
${ }^{1}$ Fouzi Harrou, Member, IEEE, ${ }^{2}$ Belkacem Khaldi \\ ${ }^{1}$ King Abdullah University of Science and Technology (KAUST) ${ }^{2}$ LESIA Laboratory, Department of Computer Science \\ CEMSE Division \\ Thuwal, 23955-6900, Saudi Arabia \\ fouzi.harrou@kaust.edu.sa \\ University of Mohamed Khider \\ R.P. 07000 Biskra, Algeria \\ khaldi.belkacem@gmail.com
}

\begin{abstract}
Fault detection plays an important role in supervising the operation of robotic swarm systems. If faults are not detected, they can considerably affect the performance of the robot swarm. In this paper, we present a robust fault detection mechanism against noise and uncertainties in data, by merging the multiresolution representation of data using wavelets with the sensitivity to small changes of an exponentially weighted moving average scheme. Specifically, to monitor swarm robotics systems performing a virtual viscoelastic control model for circle formation task, the proposed mechanism is applied to the uncorrelated residuals form principal component analysis model. Monitoring results using a simulation data from ARGoS simulator demonstrate that the proposed method achieves improved fault detection performances compared with the conventional approach.
\end{abstract}

\section{INTRODUCTION}

Swarm robotics is a new line of research that has been first founded to mimic the intelligence observed in natural swarm systems such as insects and flocks of birds [1], [2]. As with swarms in nature, while involving a large group of relatively simple robots, complex tasks in swarm robotics can be effectively accomplished collaboratively and co-operatively [3]. However, it has been reported that performing such tasks could be affected by specific faults called partial failures [4]. Therefore, the detection of such types of faults must be taken into account when designing robust controllers for robotic swarm systems.

So far, two kinds of fault detection approaches, endogenous and exogenous methods, have been developed to monitor robotics swarm systems [5]. Endogenous fault detection methods are the type of techniques in which a robot is able to detect failures arising in its own components. A few works approaching such methodology can be found in [6]-[9]. However, these methods might not be able to signal few kinds of anomalies such as dead battery to the other robots of the swarm.

On the other hand, exogenous fault detection methods are the type of methods that have recently been a matter of interest in the literature of swarm robotics. While compared to the endogenous fault detection methods, these methods have the ability to allow one or more robots to detect faults that occur in other robots of the swarm. This means that the fault detection decision is made collaboratively by generally tacking into account the information available in the swarm [10]. A number of works have been done in this vision. For example, Christensen et al. [10] reported an exogenous fault detection method inspired by the firefly behaviour. The method was able to allow real robots to detect faulty robots among operating robots. Jakimovski et al. [11] implemented an Artificial Immune System (AIS) algorithm that took its inspiration from the mechanisms of the T-Cell Receptor and intracellular signalling network, and which successfully detected faults in a robotics swarm system. In the same vision, Tarapore et al. [12] adopted an AIS technique to test the efficiency of a swarm robotics exogenous fault detection approach using a set of swarm behaviours as a case studies. In another studies, Khadidos et al. [13] suggested using a communication strategy that broadcasts the motor speeds of robots as well as their sensor readings to the neighbours in an efficient exogenous fault detection method applied to a swarm robotics system. Millard et al. [14] were able to detect faults on a robots swarm at real-time through comparing a prediction model implemented locally in each robot with the real behaviour of the other robots of the swarm.

Despite using endogenous or exogenous fault detection approaches, both can be formally developed through mathematical models or data-driven implicit models. When using mathematical-based models, the fault detection process is decided by comparing the current behaviour of the system with its predicted behaviour [15]. Unfortunately, such predicted models are usually derived from system analytical models, which are generally hard to design in a precise way [16]. Therefore, data-driven implicit models come to overcome such drawbacks and to be moreover appropriately applied in cases where explicit models cannot be used [16], [17].

In fact, data-driven implicit models are mostly approached using computational intelligence and machine learning algorithms. In this context, multivariate statistical process control schemes are among those algorithms that have been widely applied to monitor many industrial processes [18]. But, yet little works have been done to monitor robotics swarm systems. Recently, Khaldi et al. [18] were able to successfully applied a statistical procedure to monitor a group of foot-bots robots during a circle formation task [19]. 
The main idea of their procedure was to combine the flexibility of the principal component analysis (PCA) modeling to generate residuals with the greater sensitivity of the exponentially weighted moving average scheme (EWMA) to detect faults. The approach was able to detect many case studies failures on a swarm robotics system. However, EWMA-based charts ignore the multiscale nature of data, which are generally encapsulated by the noise present in measurements. This noise can easily mask the important features existed in data and therefore results in misleading indications. Moreover, noisy measurements are swiftly spread in the time-frequency domain. Thus, features should be effectively extracted from time and frequency scale. In statistics, one of the efficient tools that have this ability is the multiresolution representation of data via wavelets [20]-[23]. In this paper, an improved mechanism merging the benefits of the multiscale representation technique and EWMA scheme is proposed to deal with noisy measurements and therefore to enhance the quality of detecting faults in robot swarms. This mechanism will be called the WM-EWMA chart hereafter. Similar to the case study done in [18], the WM-EWMA approach is tested on a group of foot-bots robots performing a circle formation task via the virtual viscoelastic control (VVC) model.

\section{VIRTUAL VISCOELASTIC CONTROL MODELING APPROACH}

In the VVC model, a virtual physics approach is followed to self-organize a swarm robotics system into a circle formation [19]. The idea behind the VVC model is to model the interactions between the robots, and hence the movement of the overall swarm, using virtual viscoelastic forces. A VVC configuration of a circle of radius $r$, formed by four foot-bot robots, is illustrated in Fig. 1 .

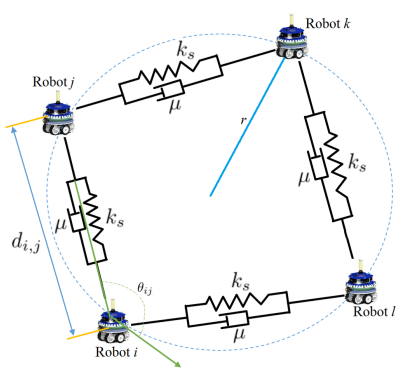

Fig. 1. A VVC configuration of a circle formed by 4-robots.

Equation (1) indicats how the total virtual viscoelastic force $F_{i}^{v v c}$ exerted on a robot $i$ is gotten. Then basing on that force, the desired forward speeds $v_{r i}$ and $v_{l i}$ of the right and the left wheels of the robot is given in equation (2).

$$
\begin{gathered}
F_{i}^{v v c}=\sum_{j=1}^{n}\left(k_{s}\left(d_{i, j}-d_{0}\right) \hat{d}_{i j}+\mu\left(v_{i}-v_{j}\right),\right. \\
{\left[\begin{array}{c}
v_{l_{i}} \\
v_{r_{i}}
\end{array}\right]=\left[\begin{array}{cc}
1 & \frac{b}{2} \\
1 & \frac{-b}{2}
\end{array}\right]\left[\begin{array}{c}
v_{i} \\
\omega_{i}
\end{array}\right],}
\end{gathered}
$$

with

$$
\omega_{i}=k_{\omega} \angle F_{i}^{v v c}, v_{i}=\frac{v_{\max }}{\sqrt{\left|\omega_{i}\right|+1}}
$$

Where in equation (1), $d_{0}=2 r \sin (\pi /(n+1))$ is the equilibrium length of the spring, $n$ is the number of neighbors, $k_{s}$ is the spring constant, $d_{i, j}$ is the current length of the spring between two interacting robots, $\hat{d}_{i j}$ is the unit vector indicating the direction of the virtual viscoelastic force, and $\mu$ is the damping coefficient. $v_{i}$ and $v_{j}$ are the velocities of the interacting robots $i$ and $j$.

Whereas in equation (2), $b$ is the distance between the robot' wheels, $\omega_{i}$ is the robot' angular velocity, and $v_{i}$ is the robot' forward speed.

Finally in equation (3), $k_{\omega}$ is a gain constant, $\angle F_{i}^{v v c}$ refers to the angle formed by the force $F_{i}^{v v c}$, and $v_{\max }$ is the maximum allowed forward speed.

In a foot-bot robot, the VVC model is achieved using the robot' RAB device (range and bearing sensing and communication device). With this device and within a maximum range $D_{r}$, messages can be sent and received to (from) neighboring robots. The constants used for the implementation of the VVC model in the foot-bot robot can be found in [19]. The implementation of the VVC model on each foot-bot is tested in the the ARGoS simulator [24]. The ARGoS-based setup of the model is further detailed in Section IV.

\section{PRoposed DETECTION STRATEGy FOR A ROBOT SWARM SUPERVISION}

The proposed monitoring mechanism is based on the PCA-based feature extraction, which is used to create the residuals for fault detection. The integrated EWMA with multiresolution data decomposition, WMEWMA scheme, is applied to residuals for monitoring the operation of a robot swarm (Figure 2). The detection mechanism is introduced in this section.

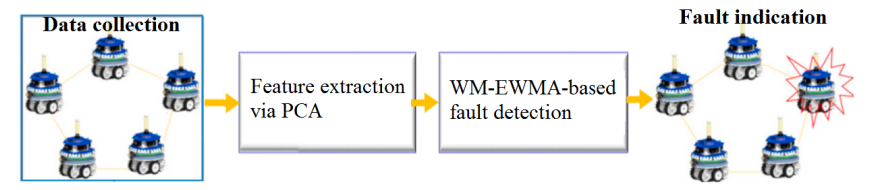

Fig. 2. Proposed mechanism for monitoring the operation of a robot swarm.

\section{A. Feature extraction via PCA}

PCA has become a well-reputed modeling procedure used for extracting relevant information from multivariate data [17], [25]-[27]. Let $\mathbf{X}=\left[\mathbf{x}_{1}^{T}, \ldots, \mathbf{x}_{n}^{T}\right]^{T} \in R^{n \times m}$ be a scaled measurement matrix with $n$ measurements and $m$ variables. The data matrix $\mathbf{X}$ is factorized using PCA scheme into two orthogonal parts,

$$
\mathbf{X}=\mathbf{T} \mathbf{W}^{T}=\sum_{i=1}^{k} t_{i} w_{i}^{T}+\sum_{i=k+1}^{m} t_{i} w_{i}^{T}=\widehat{\mathbf{X}}+\mathbf{E}
$$


where $\widehat{\mathbf{X}}$ is the approximated data and $\mathbf{E}$ is a residual data.

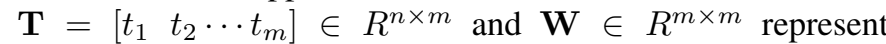
a matrix of the transformed uncorrelated variables (principal components) and loading matrix, respectively. Given certain correlation (redundancy) in data $\mathbf{X}$, the first ' $k$ ' PCs (where $k<m)$ are abale to capture most of the variability in $\mathbf{X}$. The loading matrix is usually computed via SVD of the covariance matrix $\mathbf{S}$ of the input data $\mathbf{X}$ as:

$$
\mathbf{S}=\frac{1}{n-1} \mathbf{X}^{T} \mathbf{X}=W \Lambda W^{T} \text { with } W W^{T}=W^{T} W=I_{n} .
$$

Here, $\Lambda=\operatorname{diag}\left(\sigma_{1}^{2}, \ldots, \sigma_{m}^{2}\right)$ is a diagonal matrix comprising the decreasingly ordered eigenvalues of $\mathbf{S}$ [28]. The eigenvalues $\lambda_{i}$ are equal to the variance of the $\mathrm{PC} t_{i}, \sigma_{i}^{2}$. Here, we use CPV procedure to select the number of retained PCs, $C P V(l)=\frac{\sum_{i=1}^{k} \lambda_{i}}{\sum_{i=1}^{m} \lambda_{i}} \times 100$. In this CPV procedure, the number of PCs is determined by counting PCs until the cumulative variance explains the desired percentage (e.g., 90\%) of the total variance.

In [18], a data-driven approach based on PCA model for monitoring a robot swarm has been developed. In this approach, a PCA model was employed for generating residuals and an EWMA chart was used to detect faults in the monitored robot swarm. However, the noise present in measurements can mask important features in data or lead to misleading indications by increasing false alarms and missed detections. In addition, EWMA-based charts don't consider the multiscale nature of data. Robustness of a monitoring system is crucial to operating in noisy environments whilst preserving sensitivity to anomalies. The measurement noise is spread in time-frequency domain which needs suitable feature extraction from both time and frequency scale. Wavelet-based multi-scale representation of data has shown to be a powerful data analysis tool since it can extract useful data simultaneously from time as well as frequency domain. To cope with the aforementioned limitations, we propose an approach based on the fusion of the EWMA scheme with wavelet-based multiscale analysis for further enhanced detection results.

\section{B. The WM-EWMA approach}

Integrating the benefits of multiscale representation with those of the EWMA approach should result in an enhanced monitoring system. To achieve this integrated approach, we developed the WM-EWMA-based fault detection scheme based on the wavelet coefficients at different scales.

The EWMA procedures have been largely applied to monitor the operation of industrial processes [29], [30]. The EWMA decision rule is computed by [31],

$$
z_{t}=\nu x_{t}+(1-\nu) z_{t-1}, \quad t=1,2, \ldots, \quad 0<\nu \leq 1,
$$

where $z_{0}$ is the fault-free mean of data, $\mu_{0}$, and $\nu$ is the smoothing parameters. When the charting statistic, $z_{t}$, becomes larger than a threshold, $U C L$, a signal of a potantial fault is triggered.

$$
U C L=\mu_{0}+3 \sigma_{0} \sqrt{\left(\frac{\nu}{(2-\nu)}\left[1-(1-\nu)^{2 t}\right]\right.},
$$

where $\sigma_{0}$ is standard deviation of the fault-free data.

It should be pointed out that the presence of highly noisy measurements negatively affects the performance of a fault detection procedure. The effect of noise has to be handled such that the faults could be detected successfully. Since the measurement noise is multi-scale in nature, a multi-scale representation using wavelets have been used for detection. Here, we design an innovative fault detection methodology by integrating a PCA model with WM-EWMA monitoring scheme for enhanced monitoring capability of swarm robotics systems.

Multiresolution representation of signal using wavelet permits simultaneous time and frequency analysis of a signal. By using the Discrete Wavelet Transform (DWT) a signal, $x(t)$, can be expressed as a combination of approximation, $A_{J}(t)$ and detail coefficients, $D_{j}(t)[32]$.

$$
x(t)=A_{J}(t)+\sum_{j=1}^{J} D_{j}(t),
$$

where the coarsest scale $J$ usually termed the decomposition level.

We applied EWMA anomaly detection method to detailed signals obtained using a multiscale representation of the data. A fault-free training data set was utilized to compute the control limits at multiple scales, which were then applied to the detailed signals of the testing data on multiple scales when these thresholds are surpassed, the presence of a fault is indicated. This proposed monitoring scheme is diagrammatically summarized in Figure 3.

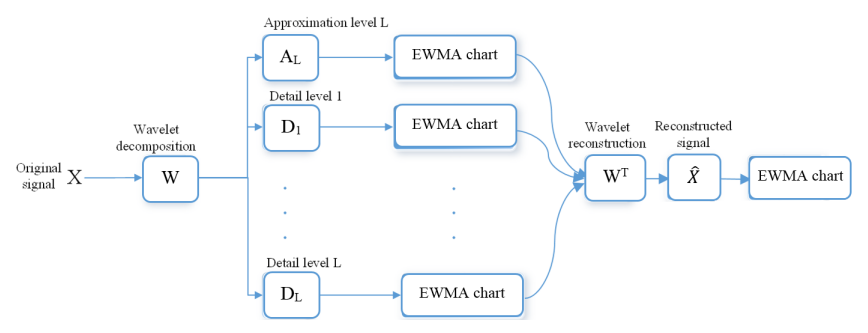

Fig. 3. Multiscale EWMA monitoring chart.

\section{Combination of PCA and WM-EWMA scheme for fault detection:}

In PCA modeling, the few retained principal components (contain the most relevant information) capture the largest amount of covariance in the data while the smaller PCs represent the residuals of the process. These residuals become closer to zero under the normal conditions, whereas a larger residuals values are obtained in the presence of abnormal conditions. Unfortunately, in a noisy environment, faults and important features can easily get lost in the noise. In other words, a large amount of noise can mask faults, and ultimately influence fault detection capability. Residuals from PCA are used as the input to fed WM-EWMA approach for detecting 
potential faults in a swarm robot. We can evaluate the process performance by applying the WM-EWMA chart to the generated residuals from the PCA model. The novelty of our approach is to develop a robust fault detection approach to noise measurements by exploiting the benefits of the multiscale representation of data and those of the EWMA scheme to better detect abnormal behaviors. Figure 4 schematically presents the proposed approach. To monitor the operating conditions of the inspected swarm robot, we first construct an appropriate PCA model using fault-free measurements and then compare the predicted from the model with measured (simulated) data. And fault detection is performed by evaluating the generated residuals based on the WM-EWMA monitoring scheme.

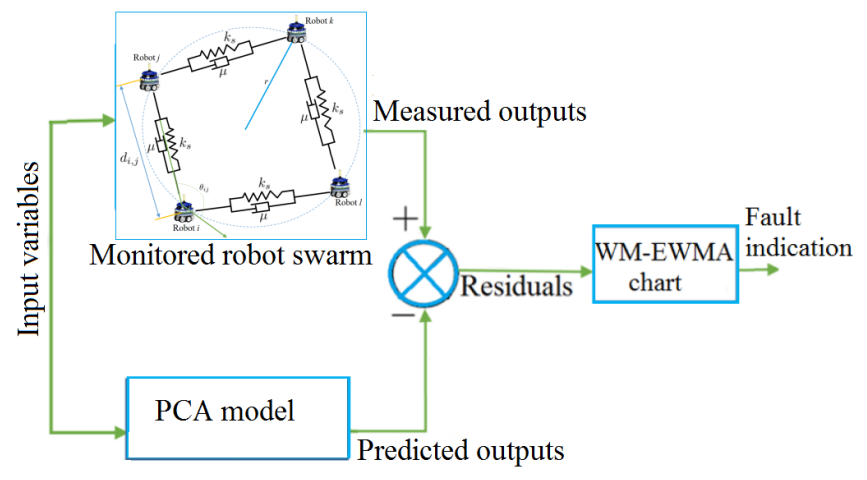

Fig. 4. A diagrammatic representation of the proposed procedure.

\section{Simulation Results}

In this study, a circle formation task is achieved using an ARGoS-based implementation of the VVC model in a swarm of 6 foot-bots. The ARGoS configuration setup of the undergoing simulations is as follows:

The foot-bots are randomly distributed in an indoor area of $10 * 6 m^{2}$ of surface. The RAB device of each foot-bot is activated within a range $D_{r}=3 \mathrm{~m} .5$ runs of 1500 time step each are performed for the same experiment. During the experimental simulations, the data in Table.I are collected to be further used as inputs/outputs for the proposed monitoring methodology.

TABLE I

MEASUREMENTS OBTAINED FROM THE ARGOS SIMULATOR.

\begin{tabular}{l|l}
\hline Parameter & Description \\
\hline$v_{r_{i}}$ & Right Wheel Forward Speed \\
\hline$v_{l_{i}}$ & Left Wheel Forward Speed \\
\hline$F_{i}^{v v c}$ & Virtual Viscoelastic Force length \\
\hline$\angle F_{i}^{v v c}$ & Virtual Viscoelastic angle \\
\hline
\end{tabular}

The training data collected in the absence of abnormalities would be used for building the PCA model and the developed model would be used for validating the testing data by the use of the conventional EWMA chart and its improved version WM-EWMA approach. The training data set consists of 3000 samples and 12 variables (i.e., viscoelastic force-length and viscoelastic force angle from each robot). CPV approach is employed to determine the number of PCs in the PCA model. Three PCs, which capture $96 \%$ of the useful information in the inspected swarm, are selected in order to build the PCA model. Once the model is developed from the training data, it would be used with the proposed WM-EWMA approach to detect anomalies in the testing data. Four types of anomalies in robotic swarm systems, namely bias anomaly, intermittent, random walk, and complete stop faults would be introduced into the testing data.

\section{A. Detection results}

The faults types considered in this study have been injected to the true measures of the motor and the RAB sensor of one or more foot-bots while a swarm of six foot-bots is performing a circle formation task. All partially faulty data were injected in real time during an ARGoS based simulation of the desired task.

1) Cases of a robot swarm with abrupt faults: To verify the effectiveness of the proposed approch in detecting abrupt faults in a robot swarm, we introduced a bias to the measurements of the viscoelastic force-length of the first robot from sample times 150 to 200. The amplitude of the introduced bias is equal to $10 \%$ of the total variation found in the raw data. The performances of conventional EWMA chart and its multiscale extension, WM-EWMA, in detecting a fault in swarm robots are studied under noisy environment. To do so, let us consider scenarios when the measurement sensors were corrupted by noise. We modeled measurements noise with Gaussian distribution with zero mean and a standard deviation, $\sigma$, but this time we investigate different values of $\sigma=0.3,0.9,1.7$. The results of the two charts for testing data with $\sigma=0.9$ (moderate noisy data)are demonstrated in Figures 5.
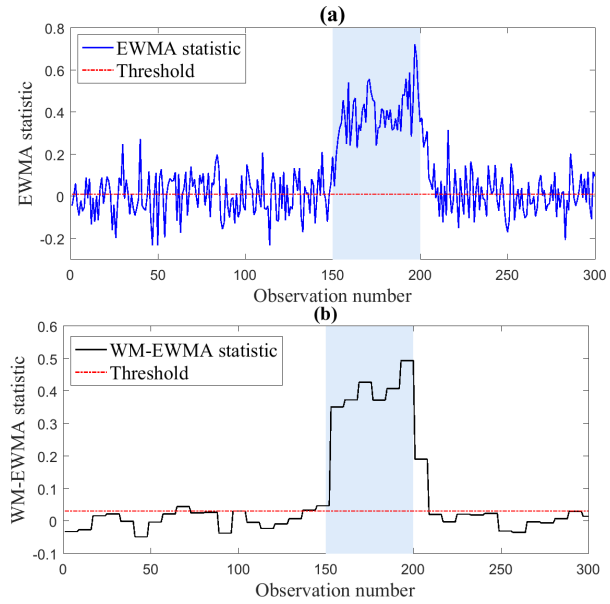

Fig. 5. (a) EWMA and (b) WM-EWMA schemes in the case of an abrupt fault, moderate noisy data example.

To make statistically valid conclusions on the robustness of these two monitoring charts against noise measurements, 
a Monte Carlo simulation with 1000 realizations is accomplished in each scenario (Table II). Table II confirms the robustness to noise measurements and sensitivity to abrupt faults of the proposed PCA-based WM-EWMA approach compared to the conventional approach.

TABLE II

FAR AND MDR VALUES OF THE EWMA AND MW-EWMA SCHEMES IN VARIOUS SCENARIOS OF NOISY DATA.

\begin{tabular}{|c|c|c||c|c|}
\hline \multicolumn{3}{|c|}{ EWMA chart } & \multicolumn{2}{c|}{ WM-EWMA chart } \\
\hline$\sigma$ & FAR & MDR & FAR & MDR \\
\hline 0.3 & 7.33 & 1.49 & 0.33 & 0.80 \\
\hline 0.9 & 44.20 & 2.25 & 6.96 & 1.95 \\
\hline 1.7 & 74.70 & 9.45 & 9.44 & 2.47 \\
\hline
\end{tabular}

2) Cases of a robot swarm with intermittent faults: Here, the robustness to sensor noise and sensitivity to intermittent faults of the proposed approach have been investigated. An intermittent fault has been introduced into the raw data, with a bias of amplitude $40 \%$ of the total variation of raw measurements from time instant 50 to 100 and bias of amplitude $10 \%$ for time instant 150 to 200. Figures 6 illustrate the monitoring results of EWMA and WM-EWMA approaches for the moderate noise level case in which the raw data are tainted with moderate noisy data $(\sigma=0.9)$. Table III summarize the performance of the two approaches in term of the average of FAR and MDR computed using a Monte Carlo simulation with 1000 replicated simulations. Results in Table III confirms the superiority of the WM-EWMA approach compared to conventional EWMA approach shows that the proposed approach results in lowest FAR and MDR compared to the conventional one.
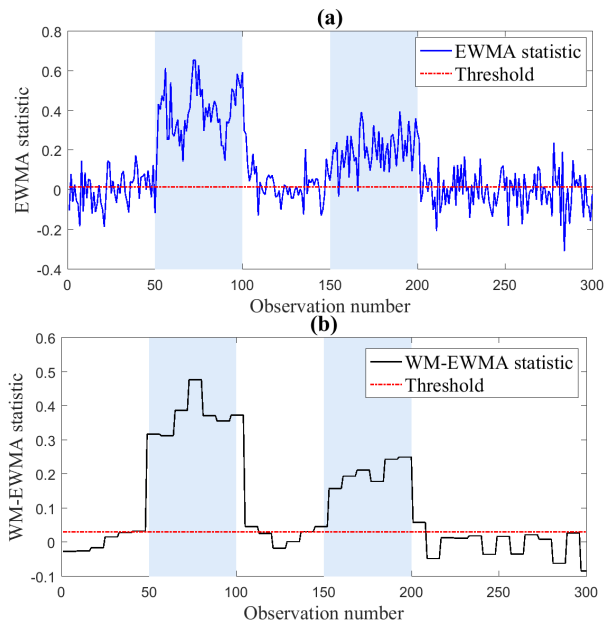

Fig. 6. (a) EWMA and (b) WM-EWMA schemes in the case of an intermittent fault, moderate noisy data example.

In summary, accurate and timely detection of faults in a robot swarm is important to avoid deterioration and degradation of the efficiency of the monitored swarm. The presence of noisy data degrades the capability of a fault
TABLE III

FAR AND MDR VALUES OF THE EWMA AND MW-EWMA SCHEMES IN VARIOUS SCENARIOS OF NOISY DATA.

\begin{tabular}{|c|c|c||c|c|}
\hline & \multicolumn{3}{|c|}{ EWMA chart } & \multicolumn{2}{c|}{ WM-EWMA chart } \\
\hline$\sigma$ & FAR & MDR & FAR & MDR \\
\hline 0.3 & 10.70 & 2.40 & 1.20 & 0.90 \\
\hline 0.9 & 49.10 & 4.38 & 8.96 & 3.20 \\
\hline 1.7 & 78.15 & 14.91 & 12.50 & 7.11 \\
\hline
\end{tabular}

detection system. Regretfully, the conventional PCA-EWMA is not entirely reliable, that is, it produces several false alarms and potential faults could be missed in the presence of noisy data. A proper integration of EWMA approach together with a wavelet-based multiscale representation is proposed to keep a suitable sensitivity to faults whilst reducing the influence of measurements noise. Although the proposed approach is successful in detecting of different faults in a robot swarm under noisy environment.

\section{CONClusion}

This work proposes an improved EWMA scheme applied to a robotic system for fault detection under noisy conditions. This method combines the advantages of PCA, the EWMA chart, and wavelet-based multiresolution smoothing. PCA model is used to generate residuals for fault detection. The new detection scheme, WM-EWMA, merges the benefits of multiscale representation of data and those of the EWMA scheme to suitably detect faults. Here, multiscale representation of data using wavelets, which is able to decouple features/noise, has been used to enhance fault detection performance minimizing uncertainties and noise effects. Superiority and robustness of the proposed approach to noise measurements compared to that of the conventional PCA-EWMA have been tested by simulated data obtained from the ARGoS simulator.

\section{ACKNOWLEDGEMENT}

This publication is based upon work supported by the King Abdullah University of Science and Technology (KAUST) Office of Sponsored Research (OSR) under Award No: OSR-2015-CRG4-2582. The work is done in collaboration with the LESIA Laboratory, Department of Computer Science, University of Mohamed Khider, Biskra, Algeria.

\section{REFERENCES}

[1] B. Khaldi and F. Cherif, "An overview of swarm robotics: Swarm intelligence applied to multi-robotics," International Journal of Computer Applications, vol. 126, no. 2, 2015.

[2] Y. Tan and Z. Zheng, "Research advance in swarm robotics," Defence Technology, vol. 9, no. 1, pp. 18-39, 2013.

[3] B. Yang, Y. Ding, Y. Jin, and K. Hao, "Self-organized swarm robot for target search and trapping inspired by bacterial chemotaxis," Robotics and Autonomous Systems, vol. 72, pp. 83-92, 2015.

[4] J. D. Bjerknes and A. F. Winfield, "On fault tolerance and scalability of swarm robotic systems," in Distributed autonomous robotic systems. Springer, 2013, pp. 431-444. 
[5] H. Lau, "Error detection in swarm robotics: A focus on adaptivity to dynamic environments," 2012.

[6] E. Skoundrianos and S. Tzafestas, "Finding fault-fault diagnosis on the wheels of a mobile robot using local model neural networks," IEEE Robotics \& Automation Magazine, vol. 11, no. 3, pp. 83-90, 2004.

[7] A. Christensen, R. OǴrady, M. Birattari, and M. Dorigo, "Fault detection in autonomous robots based on fault injection and learning," Autonomous Robots, vol. 24, no. 1, pp. 49-67, 2008.

[8] A. Christensen, R. O'Grady, M. Birattari, and M. M. Dorigo, "Automatic synthesis of fault detection modules for mobile robots." in AHS, 2007, pp. 693-700.

[9] M. Mokhtar, R. Bi, J. Timmis, and A. Tyrrell, "A modified dendritic cell algorithm for on-line error detection in robotic systems," in IEEE Congress on Evolutionary Computation. IEEE, 2009, pp. 2055-2062.

[10] A. Christensen, R. OGrady, and M. Dorigo, "From fireflies to fault-tolerant swarms of robots," IEEE Transactions on Evolutionary Computation, vol. 13, no. 4, pp. 754-766, 2009.

[11] B. Jakimovski and E. Maehle, "Artificial immune system based robot anomaly detection engine for fault tolerant robots," in International Conference on Autonomic and Trusted Computing. Springer, 2008, pp. $177-190$.

[12] D. Tarapore, P. Lima, J. Carneiro, and A. Christensen, "To err is robotic, to tolerate immunological: fault detection in multirobot systems," Bioinspiration \& biomimetics, vol. 10, no. 1, p. 016014, 2015.

[13] A. Khadidos, R. Crowder, and P. Chappell, "Exogenous fault detection and recovery for swarm robotics," IFAC-PapersOnLine, vol. 48, no. 3, pp. $2405-2410,2015$.

[14] A. Millard, J. Timmis, and A. Winfield, "Run-time detection of faults in autonomous mobile robots based on the comparison of simulated and real robot behaviour," in 2014 IEEE/RSJ International Conference on Intelligent Robots and Systems. IEEE, 2014, pp. 3720-3725.

[15] F. Harrou, L. Fillatre, and I. Nikiforov, "Anomaly detection/detectability for a linear model with a bounded nuisance parameter," Annual Reviews in Control, vol. 38, no. 1, pp. 32-44, 2014.

[16] V. Venkatasubramanian, R. Rengaswamy, S. N. Kavuri, and K. Yin, "A review of process fault detection and diagnosis: Part iii: Process history based methods," Computers \& chemical engineering, vol. 27, no. 3, pp. 327-346, 2003.

[17] S. Yin, S. Ding, X. Xie, and H. Luo, "A review on basic data-driven approaches for industrial process monitoring," IEEE Transactions on Industrial Electronics, vol. 61, no. 11, pp. 6418-6428, 2014.

[18] B. Khaldi, F. Harrou, F. Cherif, and Y. Sun, "Monitoring a robot swarm using a data-driven fault detection approach," Robotics and Autonomous Systems, vol. 97, pp. 193-203, 2017.

[19] B. Khaldi and F. Cherif, "Swarm robots circle formation via a virtual viscoelastic control model," in Modelling, Identification and Control (ICMIC), 2016 8th International Conference on. IEEE, 2016, pp. 725-730.

[20] F. Harrou, Y. Sun, and M. Madakyaru, "An improved wavelet-based multivariable fault detection scheme," in Uncertainty Quantification and Model Calibration. InTech, 2017.

[21] M. Madakyaru, F. Harrou, and Y. Sun, "Improved anomaly detection using multi-scale PLS and generalized likelihood ratio test," in Computational Intelligence (SSCI), 2016 IEEE Symposium Series on. IEEE, 2016, pp. 1-6.

[22] M. Sheriff, F. Harrou, and M. Nounou, "Univariate process monitoring using multiscale Shewhart charts," in International Conference on Control, Decision and Information Technologies (CoDIT). IEEE, 2014, pp. 435-440.

[23] F. Harrou, M. N. Nounou, and H. N. Nounou, "Enhanced monitoring using pca-based glr fault detection and multiscale filtering," in Computational Intelligence in Control and Automation (CICA), 2013 IEEE Symposium on. IEEE, 2013, pp. 1-8.

[24] C. Pinciroli, V. Trianni, R. OŠGrady, G. Pini, A. Brutschy, M. Brambilla, N. Mathews, E. Ferrante, G. D. Caro, and F. Ducatelle, "ARGoS: a modular, parallel, multi-engine simulator for multi-robot systems," Swarm intelligence, vol. 6, no. 4, pp. 271-295, 2012.

[25] S. Qin, "Survey on data-driven industrial process monitoring and diagnosis," Annual Reviews in Control, vol. 36, no. 2, pp. 220-234, 2012.

[26] F. Harrou, M. Nounou, and H.N.Nounou, "A statistical fault detection strategy using PCA based EWMA control schemes," in 9th Asian Control Conference (ASCC). IEEE, 2013, pp. 1-4.
[27] R. Bro and A. K. Smilde, "Principal component analysis," Analytical Methods, vol. 6, no. 9, pp. 2812-2831, 2014.

[28] J. Jackson and G. Mudholkar, "Control procedures for residuals associated with principal component analysis," Technometrics, vol. 21, p. 341Ü349, 1979.

[29] F. Harrou, Y. Sun, B. Taghezouit, A. Saidi, and M.-E. Hamlati, "Reliable fault detection and diagnosis of photovoltaic systems based on statistical monitoring approaches," Renewable Energy, vol. 116, pp. 22-37, 2018.

[30] N. Zerrouki, F. Harrou, Y. Sun, and A. Houacine, "Accelerometer and camera-based strategy for improved human fall detection," Journal of medical systems, vol. 40, no. 12, p. 284, 2016.

[31] D. Montgomery, Introduction to statistical quality control. John Wiley \& Sons, 2007.

[32] R. X. Gao and R. Yan, Wavelets: Theory and applications for manufacturing. Springer Science \& Business Media, 2010. 